\title{
Individuality in the groans of fallow deer (Dama dama) bucks
}

\author{
D. Reby ${ }^{1}$, J. Joachim ${ }^{1}$, J. Lauga ${ }^{2}$, S. Lek ${ }^{3}$ and S. Aulagnier ${ }^{1}$ \\ ${ }^{1}$ Institut de Recherche sur les Grands Mammifères, I.N.R.A., B.P. 27, 31326 Castanet-Tolosan cedex, France; email: reby@toulouse.inra.fr \\ ${ }^{2}$ CNRS UMR 5552 LET, Bat 4R3, Université Paul Sabatier, 118 route de Narbonne, 31062 Toulouse cedex, France \\ ${ }^{3}$ CNRS UMR 5576 CESAC, Bat 4R3, Université Paul Sabatier, 118 route de Narbonne, 31062 Toulouse cedex, France \\ (Accepted 30 September 1997)
}

\begin{abstract}
Vocal signatures and individual recognition are documented in a wide range of avian and mammalian species, but little is known about cervids. However, the existence of individual characteristics in cervid vocalizations is highly probable, as the individual morphology of their vocal organ determines the spectral structure of the uttered signal. Here, we report the presence of individual characteristics in the spectral structure of fallow deer groans recorded during the rutting period. We digitized 147 vocalizations from four adult males and transformed each of them into a power spectrum of 32 values in order to represent the frequency distribution of the sound power. A neural network discrimination with cross validation performed on the resulting variables allowed us correctly to identify $87.9 \%$ of the tested vocalizations. The spectrum characteristics of an individual remained stable over the rutting period, and probably over several consecutive ruts (the vocalizations of one male recorded during a previous rutting period were also correctly classified). We therefore conclude that the groan may constitute a vocal signature. The individually structured groan may provide a valuable basis for individual recognition during the breeding season and therefore may play an important role in the social interactions observed during this period.
\end{abstract}

Key words: Dama dama, vocalization, individual calls

\section{INTRODUCTION}

The potential for individual recognition has been found in the spectral dynamics of the vocalizations of many mammalian families (spider monkeys, Ateles geoffroyi: Chapman \& Weary, 1990; timber wolves, Canis lupus: Tooze, Harrington \& Fentree, 1990; spotted hyenas, Crocuta crocuta: East \& Hofer, 1991a; mouse lemurs, Microcebus murinus: Zimmerman \& Lerch, 1993; harbour seals, Phoca vitulina: Hanggi \& Schusterman, 1994; sea otters, Enhydra lutris: McShane et al., 1995). Concerning cervids, only two studies deal with individual differences in vocalizations: McComb (1988) found individual differences in roars emitted by red deer (Cervus elaphus) stags during the rutting period. In reindeer (Rangifer tarandus), vocalizations also aid mutual individual recognition between mother and young (Epsmark, 1971, 1975).

Fallow deer bucks, like most polygynous cervid males, call loudly during the rutting period. Bucks generally groan repeatedly to females while approaching, herding or chasing them, as well as to mature and young males (Braza, Garcia \& Alvarez, 1986). The fallow deer groan is a short, low-pitched and stereotyped vocalization. It sounds guttural and comes from a vibration of the vocal cords that is probably produced during inhalation (Gilbert, 1968, pers. obs.).

Here, we analyse the acoustic structure of groans uttered by four fallow deer bucks in order to identify the presence of information concerning individuality within the signal structure.

\section{METHODS}

\section{Study area and animals}

The study was carried out in a private enclosure located $25 \mathrm{~km}$ north-east of Toulouse, France. Ninety percent of this 130 ha enclosure is wooded. The remainder consists of a 1.6 ha clearing in the middle of the enclosure and a few small glades.

The fallow deer population originates from 4 animals introduced in 1978 from a neighbouring farm. Two more individuals were imported from Scotland in 1986. The population reached 130 individuals in October 1993, including 39 fawns, 64 mature does ( $\geq 1$ year old), 20 young bucks, and 7 mature bucks ( $\geq 3$ years old). All 
but a few fawns were marked with individually recognizable collars (Vincent et al., 1996).

\section{Data collection}

We recorded groans during autumn 1993, from the onset of vocal activity (9 October) until its conclusion (2 November). We used a Realistic 3390-30 cardiod microphone fixed at the focus of a $60 \mathrm{~cm}$ parabolic reflector and a Universum CT 2183 A tape recorder with a repeatedly checked $4.75 \mathrm{~cm} / \mathrm{s}$ speed. Most of the recordings were performed during daytime in the main clearing of the enclosure. Four semi-tame mature bucks were recorded within $30-50 \mathrm{~m}$. The 3 younger bucks never groaned.

We selected 141 recordings from these bucks (we rejected vocalizations which were recorded from bucks that were either not facing the microphone or with high levels of background noise of wind or air traffic). All these groans were taken from different bouts. They were unequally distributed: 60 groans for buck A (from 11 October until the 2 November), 26 for buck B (from 9 until 12 October), 14 for buck C (on 15 October), and 41 for buck D (from 11 until 17 October). In addition, 6 groans from buck A recorded in October 1992 were considered.

\section{Sound analysis}

We digitized vocalizations using an analogical/digital converter (Mac Recorder ${ }^{\mathrm{TM}}$ hardware, and Sound Edit $^{\mathrm{TM}}$ software). Groans were first digitized at a sampling rate of $22.3 \mathrm{kHz}$ and spectrograms of each vocalization were edited with Sound Edit ${ }^{\mathrm{TM}}$ in order to estimate the spectral dynamics of each vocalization. As above $5 \mathrm{kHz}$ the signal structure disappeared behind low level background noise in most vocalizations, we chose to re-digitize the vocalizations with an $11 \mathrm{kHz}$ sampling rate ( 8 bit amplitude resolution). The few signals which contained energy above the $5.5 \mathrm{kHz}$ Nyquist frequency were automatically filtered by the converter in order to prevent aliasing.

Temporal features (signal duration, syllabic structure) were directly measured on the waveform. As the first step of the assessment of the inter-individual differences, intermediate band spectrograms $(176.5 \mathrm{~Hz}$ analysis filter bandwidth) provided representations for visual comparison in the time frequency domain.

Using a program written in Pascal by J. Lauga (Joachim \& Lauga, 1996), we performed a Fast Fourier Transform (FFT size $=64$, overlap $=50 \%$, frequency resolution $=173.9 \mathrm{~Hz}$, Hanning window function) on the total length of the signal in order to calculate the Average Power Spectrum (APS) of each vocalization. The transform output generated 32 variables, which represent the distribution of sound energy over the duration of the signal for each of 32 intervals of 173.9 $\mathrm{Hz}$ (the power spectrum is normed on a total amount of
1000 and transformed in $\mathrm{dB}$ ). These 32 variables depict the sound energy distribution in the frequency domain over the total length of the signal, likely to provide a good representation of the spectral features. Such a computation eliminates the temporal size of the signal.

\section{Statistical analysis}

We performed several 1-factor ANOVAs on the different temporal measurements, in order to assess intraor inter-individual differences of these parameters. Then, we analysed variability of the 32 APS values within and between individuals on the sample of 141 vocalizations. For this, we performed a multivariate analysis of variance (1-factor MANOVA, tested factor: the individual) on the APS set, and univariate analysis of variance (1-factor ANOVA) on each of the 32 APS variables. All analyses of variance tests were processed using SYSTAT (Systat, 1992), and significance was accepted at an alpha level of 0.05 .

We then performed a supervised clustering by neural networks (Reby et al., 1997) based on the back propagation gradient principle (Rumelhart, Hinton \& Williams, 1986). Data were processed in 2 stages. The first stage consisted of training the network to establish a vocalization model for each individual, with all the 141 vocalizations recorded in 1993. As in discriminant analysis, the first result is the percentage of correct classifications of the training set vocalizations (resubstitution test). In the second stage, we tested the model's strength by performing 5 cross-validation tests. This evaluated the model's ability to classify additional vocalizations. For this purpose, we trained a neural network with a sample consisting of two-thirds of each individual's vocalizations $(\mathrm{N}=105)$. This network was then tested with its respective validation set comprising the remaining third of vocalizations $(\mathrm{N}=36)$. This operation was repeated 5 times with different random samples so that our results are mean scores over 5 classification tests.

In order to assess the stability of the individuality from one year to the next, another validation test was performed (on the basis of the network trained with the 141 vocalizations from 1993), with 6 vocalizations from buck A recorded in 1992.

\section{RESULTS}

\section{Time structure}

Groan duration ranged between 0.19 and $0.65 \mathrm{~s}$ $(\bar{x} \pm$ S.D. $=0.36 \pm 0.07 \mathrm{~s} ; \mathrm{N}=141)$. There were no significant differences between individuals in this duration measure $\left(F_{3,137}=1.51 ; P=0.215\right)$. Groans were typically composed of a series of $5-22 \quad(\bar{x} \pm$ S.D. $=12.3 \pm 3.1$ $\mathrm{N}=137$ ) regularly spaced 'pulses' (Fig. 1). These pulses are the vibration of the vocal cords, and constitute the fundamental frequency of the call. It ranged from 21.2 to 71.0 pulses $/ \mathrm{s}(\overline{\mathrm{x}} \pm$ S.D. $=34.8 .2 \pm 8.8 \mathrm{pulses} / \mathrm{s}$, 


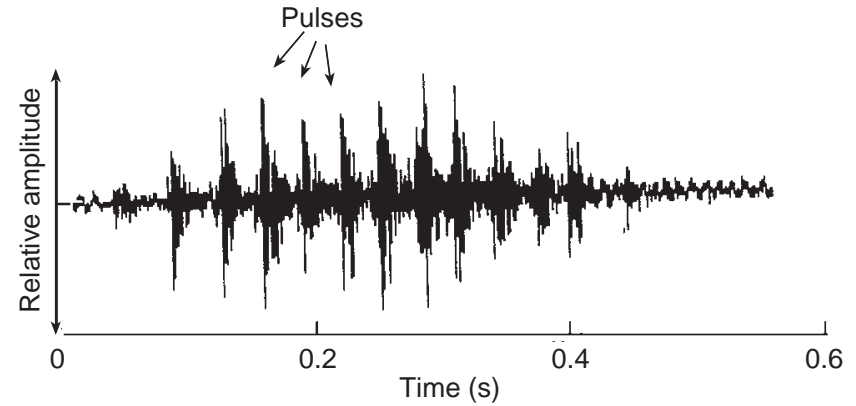

Fig. 1. Waveform of a typical fallow deer groan. The groan is composed of a series of pulses.

$\mathrm{N}=137$ ). In buck $\mathrm{D}$, it increased significantly from $\overline{\mathrm{X}} \pm$ S.D. $=27.6 \pm 5.2$ pulses $/ \mathrm{s} \mathrm{N}=10$ on the 11 October, to $\overline{\mathrm{x}} \pm$ S.D. $=57.6 \pm 9.5, \quad \mathrm{~N}=9$ on the 17 October $\left(F_{1,17}=74.62 ; P<0.001\right)$. Four groans of this buck recorded on this last day were so noisy that we could not analyse their temporal structure.

\section{Spectrograms}

The fallow deer groan is not a clear, tonal vocalization: it is a harsh and noisy call, the fundamental frequency is so low that the harmonic series is difficult to pick out (Fig. 2), and the clearest feature on the spectrogram is the formantic structure (assuming that a formant is defined as a range of frequencies in which there is an absolute or relative maximum in the sound spectrum, A.N.S.I., 1995). A comparison of representative spectrograms from the four bucks is given in Fig. 3. Buck D spectrograms showed a considerable degradation of the temporal structure with time, while the formants' features were more stable. Visual comparison of the spectrograms confirms that, though the differences were not sufficiently marked to provide an accurate visual classification, the features of the formants varied between individuals. Formantic structure was therefore likely to carry information on individuality.

\section{Average Power Spectrum (APS)}

The APS is a global description of the energy distribution in terms of frequency, providing a good representation of the spectral components (Fig. 4). The relation between the spectrogram and the APS is obvious: clear horizontal bands on the spectrogram correspond to minima on the spectrum, and dark horizontal bands correspond to maxima. Results of multivariate analysis showed that the sets of 32 APS values are highly significantly different between individuals (Pillai trace $=2.233 ; F_{96}, 324=9.818, P<0.001$ ). Univariate $F$ test results are shown in Table 1. All variables were significantly different between groups of vocalizations (individuals) except APS variables 12 and 13, which correspond to frequencies ranging between 1.9 and $2.3 \mathrm{kHz}$.

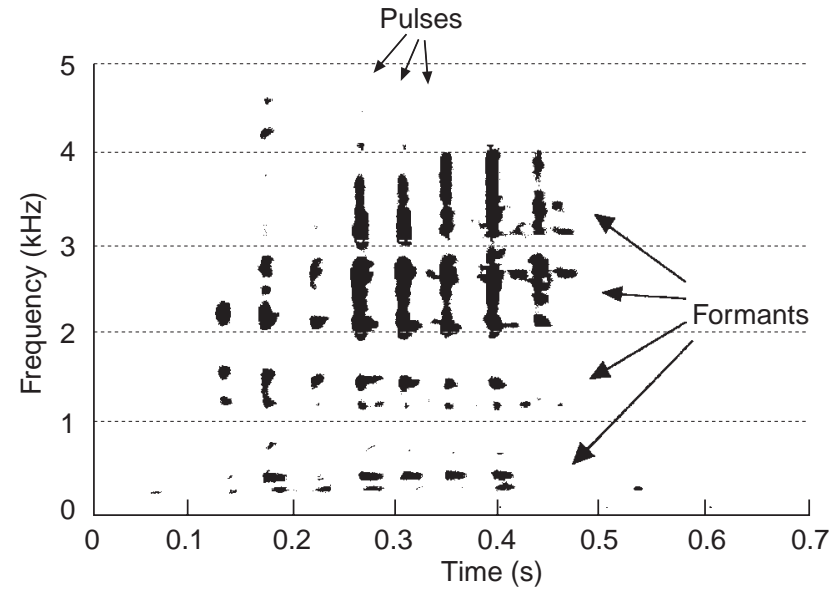

Fig. 2. Spectrogram of the same groan: temporal and formantic structures. In this example, the groan comprises 8 pulses and 4 distinct formants.

\section{Classification of vocalizations}

Performing neural network classification, the rate of correct recognition of the resubstitution test was $100 \%$. In the validation tests, the average rate of additional vocalizations that were correctly classified over the five sets was $87.9 \%$ (Table 2). In addition, the six vocalizations of buck A from 1992 were all correctly attributed.

\section{DISCUSSION}

The fallow deer groan is a complex signal with welldefined temporal and formantic structures. The analysis of the temporal features shows that, while the signal duration is not different between individuals, the pulse rate can be highly variable within one individual. Apart from the degradation of the quality of the signal across time, it is very likely that the pulse rate is highly dependent on the rate at which air is inhaled, and therefore correlated with the buck's activity.

The 32 APS variables are a good representation of the features of the formants because, in the fallow deer groan, the spectral components change only slightly over the signal duration. Multivariate analysis of variance and neural network resubstitution tests performed on these values confirmed the existence of highly significant differences between the groans of the four bucks. Cross-validation results indicated that these spectral differences are strong enough to ascribe additional vocalizations to the particular buck with a very high percentage of correct classification. We emphasize the fact that the tested vocalizations were not used in the model training, strengthening the statistical robustness of our classification (see Tooze et al., 1990). One may point out that only four individuals were recorded. If, for probabilistic reasons, the rate of correct classification decreases with the number of individuals to recognize, these result are to be interpreted within the context of the animal's behaviour. For a deer it may 
(1) (a) Buck B

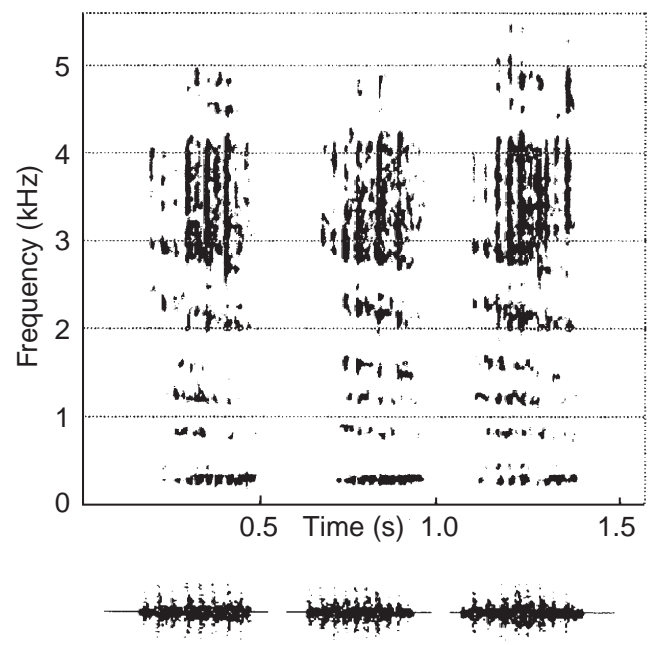

(2) (a) Buck D, beginning of vocal activity period

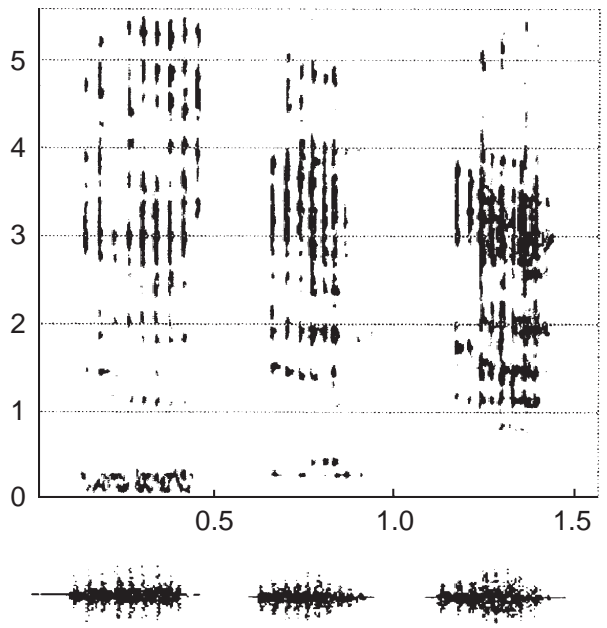

(3) (a) Buck A, beginning of vocal activity period

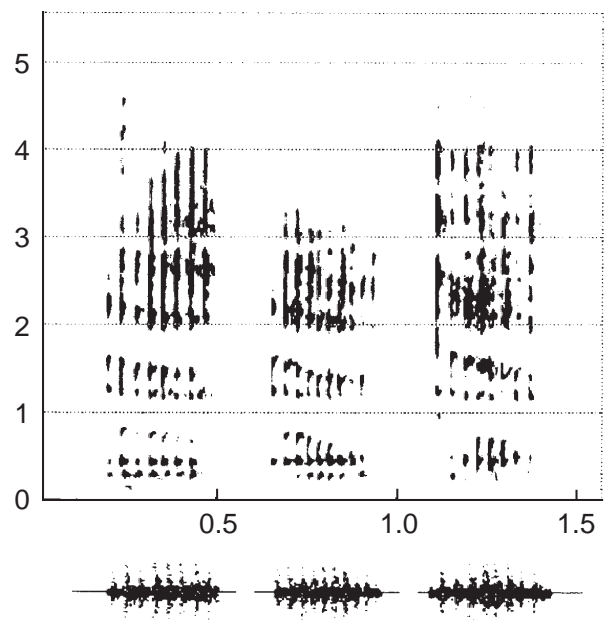

(b) Buck C

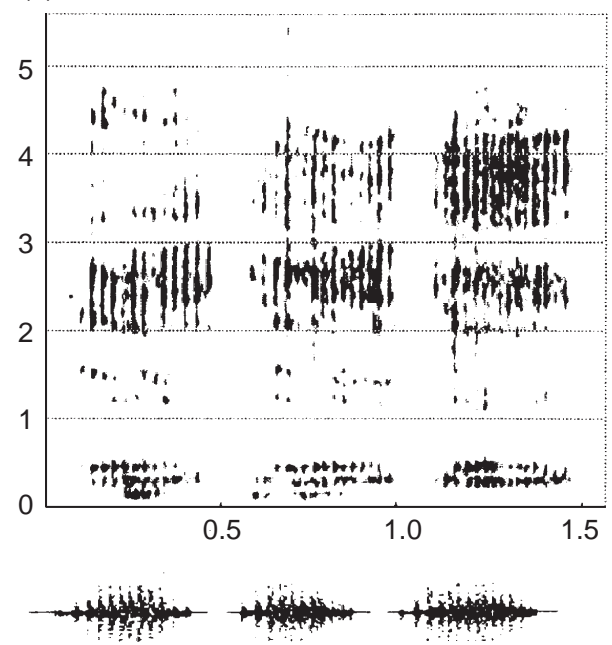

(b) Buck $D$, end of vocal activity period

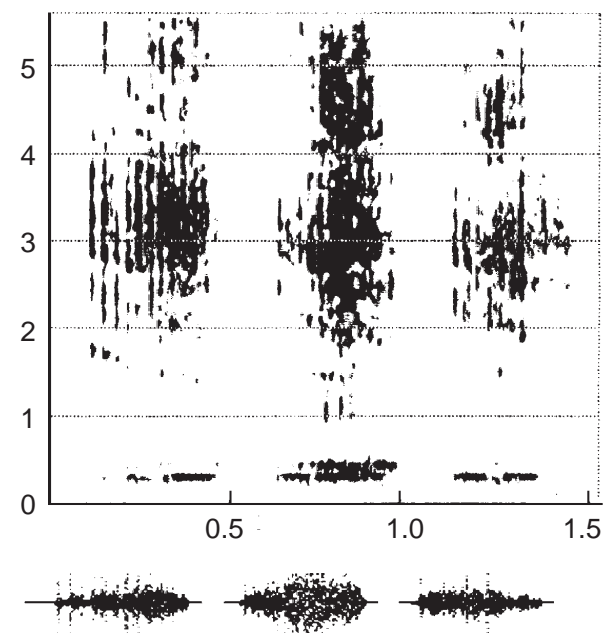

(b) Buck A, end of vocal activity period

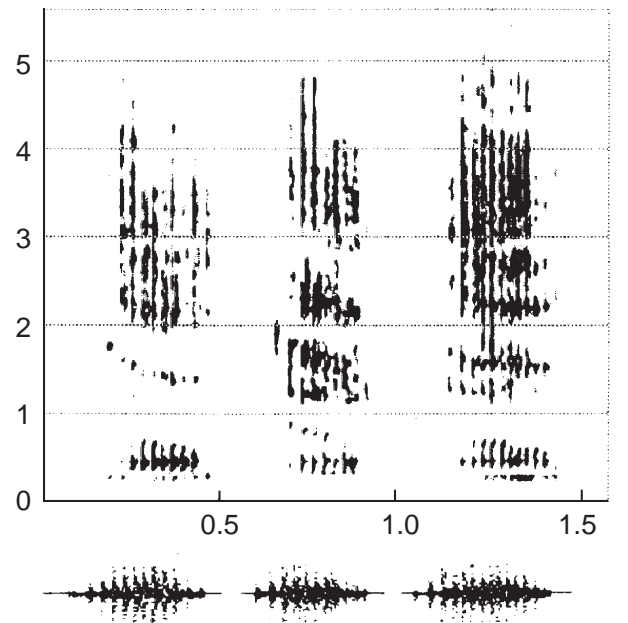

Fig. 3. (1): Waveforms and spectrograms of (a) three groans from buck B and (b) three groans from buck C. There are noticeable differences in the shape of the formants between the two individuals. (2): Waveforms and spectrograms of six groans from buck D recorded on single days (a) at the beginning (11 October) and (b) at the end (17 October) of its period of vocal activity. As the signal becomes more noisy, the pulsed temporal structure deteriorates progressively, while the formantic structure remains relatively stable. (3): Waveforms and spectrograms of six typical groans from buck A recorded at six different days during the 1993 rutting period; (a): beginning of its period of vocal activity (11, 12 and 13 October); and (b): end of its period of vocal activity (21, 28 October and 2 November). The spectrograms of the last recorded groans are more noisy, the temporal structure in the higher formants is less clearly defined. 
Table 1. Univariate F-test for each of the 32 average power spectrum variables of 141 groans from the four fallow deer bucks

\begin{tabular}{lcll}
\hline Variable(s) & Range $(\mathrm{Hz})$ & $F_{3,143}$ & $P$ \\
\hline $1-11$ & $0-1912$ & - & $*$ \\
12 & $1912-2086$ & 2.124 & $0.100 \mathrm{NS}$ \\
13 & $2086-2260$ & 2.629 & $0.053 \mathrm{NS}$ \\
$15-32$ & $2260-5062$ & - & $*$ \\
\hline
\end{tabular}

For each APS variable, inter-group $(\mathrm{N}=4)$ variance is compared to intra-group $(\mathrm{N}=141)$ variance; each group of vocalizations corresponds to one individual buck. ${ }^{*} P<0.005$

Table 2. Classification of vocalizations $(\mathrm{N}=48)$ of fallow bucks by neural network pretrained with sample vocalizations from the same bucks

\begin{tabular}{lcccccc}
\hline $\begin{array}{l}\text { Actual } \\
\text { member- } \\
\text { ship }\end{array}$ & Predicted group membership* & Total & $\begin{array}{l}\text { Correctly } \\
\text { classified } \\
(\%)\end{array}$ \\
& 1 & 2 & 3 & 4 & & \\
\cline { 2 - 6 } & 18 & 1.4 & 0 & 0.6 & 20 & 90.0 \\
1 & \pm 0.55 & \pm 0.50 & - & \pm 0.25 & & \pm 2.7 \\
2 & 0.8 & $\mathbf{8}$ & 0 & 0.2 & 9 & 88.9 \\
& \pm 0.20 & \pm 0.00 & - & \pm 0.20 & & \pm 0.0 \\
3 & 0 & 0.4 & $\mathbf{4 . 2}$ & 0.4 & 5 & 84.0 \\
& - & \pm 0.25 & \pm 0.45 & \pm 0.40 & & \pm 7.5 \\
4 & 1.2 & 0 & 0.4 & $\mathbf{1 2 . 4}$ & 14 & 88.6 \\
\hline
\end{tabular}

*Values are means of classification scores $\bar{x} \pm$ S.E. over the five tests $(\mathrm{N}=5)$

suffice merely to distinguish between familiar vocalizations and unfamiliar ones, and perhaps to discriminate between familiar individuals only.

No specific feature coding for individuality was found in the vocalization. The individuality was rather a combination of a set of spectral characteristics. This result supports Epsmark's (1975) findings which suggested that it is not usually specific features which make up the call characteristic of an individual, but rather the subtle combination and interaction of different features and the overall relationship between them. That is why it was worthwhile to look for individuality within the interaction of a great number of variables, using the APS values and a multivariate classification. The formantic structure of the vocalization remained stable in one individual from one year to the next. This stability is all the more surprising as groans are emitted only once a year and during a limited period.

Several examples in the literature suggest that, in mammals, vocalizations are probably used for individual recognition, in association with olfactive and visual cues. The fact that groaning rate is correlated with mating success in fallow deer (Clutton-Brock et al., 1988), suggests that, as in red deer (McComb, 1991), high call rate may attract females. However, individual vocal characteristics probably enable the establishment
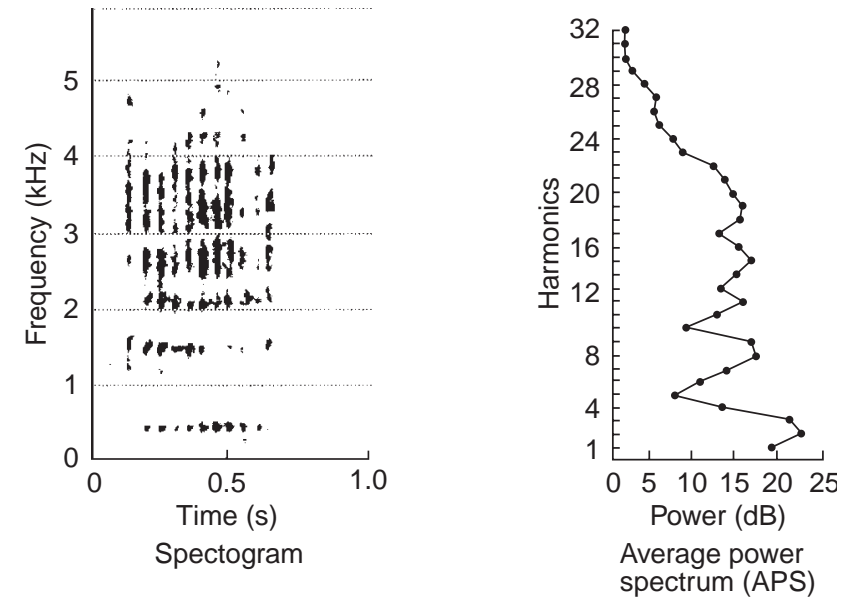

Fig. 4. Groan spectrogram (left): the sound pressure distribution (grey levels) is represented in the time-frequency domain. Average power spectrum (right) of the same roar with the 32 harmonic values (decibels); while the time dimension is suppressed it is a good quantification of the horizontal formantic structure.

of specific relationships. In other polygynous mammals, females may choose to mate with familiar males who invested most in forming an association with them (spotted hyenas: East \& Hofer 1991b; mouse lemurs: Zimmerman \& Lerch, 1993). Oestrous fallow deer females may also prefer mating with familiar males heard during the beginning of the rut or during the ruts of previous years, and they may therefore join a particular mating stand on the basis of the individual vocal characteristics of the tenant male. It is therefore possible that individually distinct vocalization, in association with reference to previous experience, may influence the reproductive success of individual bucks in a fallow deer population. Fallow deer males may also avoid engaging fights with bucks whose call is associated with an established dominance relationship, as has been suggested in other species (elephant seals: Shipley, Hines \& Buchwald, 1981; red deer: McComb, 1988; mouse lemurs: Zimmerman \& Lerch, 1993). This would be especially important at long distance, for example in closed habitat, in the absence of visual cues.

This study shows that the fallow deer groan contains information on the individuality of the caller. However, we did not study the behaviour of the other deer. Therefore, the role of individual recognition of fallow deer groans in social situations such as those discussed above remains to be demonstrated by means of playback experiments.

\section{Acknowledgements}

We thank Mr Bos, the 'Palmola enclosure' owner, who gave us the possibility of studying this population of fallow deer. We also thank Dominique Pépin and Mark Hewison who reviewed the original manuscript. 


\section{REFERENCES}

A.N.S.I. (1995). American National Standard Bioacoustical Terminology. A.N.S.I. S3.20-1995 ( ASA 114).

Braza, F., Garcia, J. E. \& Alvarez, F. (1986). Rutting behaviour of fallow deer. Acta Theriol. 31: 467-478.

Chapman, C. A. \& Weary, D. M. (1990). Variability in spider monkeys' vocalisations may provide basis for individual recognition. Am. J. Primatol. 22: 279-284.

Clutton-Brock, T. H., Green, D., Hiraiwa-Hasegawa, M. \& Albon, S. D. (1988). Passing the buck: resource defence, lek breeding and mate choice in fallow deer. Behav. Ecol. Sociobiol. 23: $281-296$.

East, L. E. \& Hofer, H. (1991a). Loud calling in a female dominated society: I. Structure and composition of whooping bouts of spotted hyenas, Crocuta crocuta. Anim. Behav. 42: 637-649.

East, L. E. \& Hofer, H. (1991b). Loud calling in a female dominated society: II. Contexts and functions of whooping of spotted hyenas, Crocuta crocuta. Anim. Behav. 42: 651-669.

Epsmark, Y. (1971). Individual recognition by voice in reindeer mother-young relationship. Behaviour 40: 295-301.

Epsmark, Y. (1975). Individual characteristics in the calls of reindeer calves. Behaviour 54: 50-59.

Gilbert, B. K. (1968). Development of social behavior in the fallow deer (Dama dama). Z. Tierpsychol. 25: 867-876.

Hanggi, E. B. \& Schusterman, R. J. (1994). Underwater acoustic displays and individual variation in male harbour seals, Phoca vitulina. Anim. Behav. 48: 1275-1283.
Joachim, J. \& Lauga, J. (1996). Populations et dialectes chez le Pinson. C. R. Acad. Sci. 319: 231-239.

McComb, K. E. (1988). Roaring and reproduction in red deer, Cervus elaphus. PhD thesis, University of Cambridge.

McComb, K. E. (1991). Female choice for high roaring rate in red deer, Cervus elaphus. Anim. Behav. 41: 79-88.

McShane, L. J., Estes, J. A., Riedman, M. L. \& Staedler, M. M. (1995). Repertoire, structure, and individual variation of vocalisations in the sea otter. J. Mammal. 76: 414-427.

Reby, D., Lek, S., Dimopoulos, I., Joachim, J., Lauga, J. \& Aulagnier, S. (1997). Artificial neural networks as a classification method in the behavioural sciences. Behav. Processes 40: $35-43$.

Rumelhart, D. E., Hinton, G. E. \& Williams, R. J. (1986). Learning representations by back-propagating error. Nature (Lond.) 323: 533-536.

Shipley, C., Hines, M. \& Buchwald, J. S. (1981). Individual differences in threat calls of northern elephant seal bulls. Anim. Behav. 29: 12-19.

Systat, Inc. (1992). Statistics, version 5.2 edition. Evanston, IL.

Tooze, Z. J., Harrington, F. H. \& Fentress, J. C. (1990). Individually distinct vocalisations in timber wolves, Canis lupus. Anim. Behav. 40: 723-730.

Vincent, J. P., Hewison, A. J. M., Angibault, J. M. \& Cargnelutti, B. (1996). Testing density estimators on a fallow deer population of known size. J. Wildl. Manage. 60: 18-28.

Zimmerman, E. \& Lerch, C. (1993). The complex acoustic design of an advertisement call in male mouse lemurs (Microcebus murinus) and sources of its variation. Ethology 93: 211-224. 